\title{
TOXINA BOTULÍNICA E HIPERIDROSE: O USO DA NEUROTOXINA NO TRATAMENTO DA DOENÇA
}

\section{REVISÃO INTEGRATIVA}

SILVA, Poliana dos Santos ${ }^{1}$

XISCATTI, Valéria Scarlleth Costa ${ }^{2}$

SOUZA, Ramon Guimarães de ${ }^{3}$

BARROSO, Wermerson Assunção ${ }^{4}$

OLIVEIRA, Adriana dos Santos ${ }^{5}$

SILVA, Poliana dos Santos. Et al. Toxina Botulínica e Hiperidrose: 0 uso da neurotoxina no tratamento da doença. Revista Científica Multidisciplinar Núcleo do Conhecimento. Ano 05, Ed. 10, Vol. 16, pp. 127-137. Outubro de 2020. ISSN: 2448-

${ }^{1}$ Graduanda em Biomedicina pela Universidade Ceuma, Campus Imperatriz, Curso de Biomedicina, Imperatriz/MA.

${ }^{2}$ Graduanda em Biomedicina pela Universidade Ceuma, Campus Imperatriz, Curso de Biomedicina, Imperatriz/MA.

${ }^{3}$ Graduado em Medicina pela Universidad Politécnica y Artística, Facultad de ciências de La Salud, Sede Ciudad Del Este, Unidade Básica de Saúde Amorim II, Zé Doca/MA.

${ }^{4}$ Doutor em Ciências pela Faculdade de Medicina da Universidade de São Paulo, Docente da Faculdade ITPAC Santa Inês, Curso de Medicina, Santa Inês/MA.

${ }^{5}$ Mestre em Patologia das Doenças Tropicais pela UFPA, Biomédica pela PUC-GO com habilitação em Patologia Clínica, Especialista em Microbiologia Clínica pela Academia de Ciência e Tecnologia São José do Rio Preto SP, Docente da Universidade Ceuma, Campus Imperatriz, Coordenação do Curso de Biomedicina, Curso de Biomedicina, Imperatriz/MA. 
0959, Link de acesso: https://www.nucleodoconhecimento.com.br/saude/uso-daneurotoxina

\section{RESUMO}

A hiperidrose é uma doença caracterizada pelo distúrbio das glândulas sudoríparas, elevando a produção de suor. O surgimento da doença se dá por diversos fatores, causando aos portadores constrangimento, problemas psicológicos, sociais e emocionais, entre outros. Um dos tratamentos mais atuais para a doença envolve o uso da toxina botulínica tipo A, largamente utilizada em diversos tratamentos médicos e estéticos. Diante disso, o presente trabalho tem como objetivo analisar a literatura acerca do tratamento da hiperidrose com a utilização da toxina botulínica, descrevendo suas propriedades terapêuticas e os mecanismos de ação para combater a doença. A pesquisa se desenvolveu por meio de revisão integrativa da literatura, de caráter exploratória, qualitativa e de natureza indutiva. Os resultados permitiram apontar que a toxina botulínica age impedindo a ativação das glândulas sudoríparas e impedindo a produção de suor, em excesso. Ainda, o tratamento é menos invasivo e mais eficaz que os demais tratamentos clínicos e cirúrgicos, e tem o benefício de apresentar poucos efeitos colaterais.

Palavras-chave: Hiperidrose, Toxina botulínica, tratamento.

\section{INTRODUÇÃO}

A hiperidrose é definida como uma condição na qual há produção de suor em excesso, para além do necessário à regulação da temperatura corporal (GUERRA NETO, 2016). Ela se apresenta na forma primária e secundária. A forma primária é caracterizada por sudorese excessiva em diversas partes do corpo, sem causa específica, e relacionada às condições fisiológicas, neuromusculares e sociais. A hiperidrose secundária, por sua vez, ocorre de maneira generalizada no corpo do indivíduo, acontece em decorrência de uma condição prévia, como hipertireoidismo, ou ainda como decorrência de efeito colateral de uma determinada medicação (HAGEMANN e SINIGAGLIA, 2019). 
Há diversos tratamentos para a hiperidrose disponíveis atualmente, mas nenhum apresenta tanta eficácia como o tratamento no qual se utiliza aplicações da toxina botulínica tipo A. Esta neurotoxina, produzida pela bactéria Clostridium botulinum, vem sendo utilizada ao longo dos anos em diversos tratamentos estéticos, de doenças e condições neuromusculares, entre estas a hiperidrose (FUJITA E HURTADO, 2018 apud AZEVEDO, 2018).

É possível notar a grande relevância que tal terapêutica vem ganhando nos últimos anos, gozando de uma grande valorização no atual contexto e sendo reconhecida como tratamento eficaz. Cabe, assim, um estudo mais aprofundado para compreender os parâmetros de atuação da toxina botulínica no tratamento da hiperidrose. Este estudo analisou a literatura relacionada ao uso da toxina botulínica tipo A no tratamento da hiperidrose, fazendo uma revisão integrativa a fim de analisar o uso e a eficácia da toxina botulínica no tratamento da hiperidrose.

\section{METODOLOGIA}

O presente trabalho foi desenvolvido por meio de uma abordagem qualitativa sobre 0 tema escolhido, utilizando-se do método indutivo para descrever as características que permeiam o tratamento da hiperidrose que utiliza a toxina botulínica. A pesquisa é do tipo exploratória, executada mediante uma revisão integrativa, feita através do levantamento bibliográfico acerca do tema, buscando-se em artigos publicados em periódicos e outras fontes escritas. Foi analisado neste trabalho o uso da toxina botulínica, o seu mecanismo de ação, a dosagem a ser ministrada e a atuação bioquímica da toxina.

$\mathrm{Na}$ revisão de literatura buscaram-se artigos indexados disponíveis nas bases de dados eletrônicas Google Acadêmico, PubMed e Portal de Periódicos Capes, publicados em língua portuguesa e inglesa entre os anos de 2010 e abril de 2020. As palavras-chave utilizadas foram as seguintes: "hiperidrose"; "toxina botulínica"; "tratamento", "sudorese". Os critérios de inclusão adotados foram: que o artigo tratasse das propriedades da toxina botulínica; que o artigo tratasse acerca da hiperidrose em relação às suas causas e tratamentos; que o artigo tratasse da toxina 
botulínica no tratamento da hiperidrose. Acessamos o texto completo de todas as referências relevantes a partir dos resultados da pesquisa. Os critérios de exclusão adotados foram: artigos que não abordassem os critérios de inclusão e que não estavam dentro do ano de publicação estipulado por essa pesquisa.

\section{RESULTADOS E DISCUSSÃO}

O estudo desenvolvido por Hagemann e Sinigaglia (2019) conceituou a hiperidrose como doença em que há a característica da produção excessiva de suor em estado ativo ou em repouso. Dos Santos e Paiva (2017) compreendem que a hiperidrose primária é capaz de atingir uma região corporal, bem como várias, não havendo uma patologia que possa ser utilizada como base para justificar tal quadro. Disto, entendese, portanto, ser uma condição cuja etiologia é desconhecida

Entendeu-se que a hiperidrose primária pode ser considerada uma doença multicausal ou de causa não especificada, podendo se dar mediante uma gama de fatores, tais como aspectos psicológicos, emocionais, sociais, hereditários e, ainda, fatores de ordem ocupacional (HAGEMANN E SINIGAGLIA, 2019).

Aferiu-se, porém, que, não obstante haja imprecisão acerca da fisiopatologia da hiperidrose, há provável relação com algum distúrbio do sistema nervoso simpático, o qual deve provocar uma hipersecreção ou hipertrofia das glândulas sudoríparas écrinas de determinadas áreas anatômicas (DOS SANTOS E PAIVA, 2017). Assim os fatores e causas supracitados poderiam refletir no funcionamento anormal das glândulas sudoríparas.

No tocante à tais glândulas, no organismo humano, estas se apresentam numa quantidade aproximada de quatro milhões, na qual um terço destas são apócrinas, e o restante é composto por glândulas sudoríparas écrinas (PINTO; MILAGRES E QUEIROZ, 2015). Foi assim, observada uma relação provável entre a hiperidrose e a diminuição das atividades de tais glândulas de maneira difusa ou a superatividade delas. 
Foi possível apontar também como fatores fisiológicos relacionados à hiperidrose, certos distúrbios anatômicos, tais como disfunções endócrinas, ou ainda, disfunções metabólicas e em último caso, disfunções neurológicas (BERNARDES et al., 2019). A análise dos resultados ainda permite aferir uma incidência considerável do fator de hereditariedade, o que se mostrou presente em estudo realizado que informou que, em 30\% a 50\% dos casos de hiperidrose, há histórico familiar relacionado à afecção (PINTO; MILAGRES E QUEIROZ, 2015).

Foi observada ainda a presença da doença na maioria de jovens, ocorrendo com frequência entre 14 a 25 anos de idade, não se observando, no entanto, com tanta frequência em idosos, provavelmente em razão da regressão espontânea das glândulas sudoríparas écrinas neste grupo de indivíduos (DOS SANTOS E PAIVA, 2017).

Os resultados também demonstraram que a hiperidrose é classificada em duas categorias, primária e secundária, sendo a forma primária relacionada à ocorrência de sudorese excessiva de maneira focal, isto é, em pontos isolados, sobretudo superfícies palmares de pés e mãos e área das axilas e face. (GUERRA NETO, 2016). A forma secundária, por sua vez, se apresenta como uma condição generalizada, na qual há a ocorrência de sudorese excessiva em mais de uma área, e ainda, apresentase relacionada à outras patologias, destacando-se entre estas patologias como obesidade e hipertireoidismo (DOS SANTOS E PAIVA, 2017).

Observou-se na literatura analisada um entendimento comum de que a hiperidrose não é considerada uma doença grave. No entanto, suas implicações psicoemocionais e sociais são profundas. Com efeito, não obstante traga riscos de ordem fisiológica ao paciente, é uma condição que degrada a qualidade de vida, sobretudo no aspecto social (CHARELLO E DUTRA, 2018).

Os resultados ainda permitiram apontar que a hiperidrose faz emergir fobias sociais, e ainda gera enorme desconforto ao indivíduo que possui a condição (BERNARDES et al., 2019), evidenciando que em certos casos, mesmo atos simples como apertos de mãos se tornam causa de depreciação de relações sociais do indivíduo (BASTOS 
et al., 2016). Demonstra-se que a hiperidrose, embora não seja letal, apresenta potencial de deterioração do convívio do indivíduo

\subsection{OS TRATAMENTOS ATUALMENTE DISPONÍVEIS PARA A HIPERIDROSE}

No tocante aos tratamentos disponíveis para a hiperidrose, foi possível categorizar estes em dois tipos: cirúrgicos e clínicos. Os tratamentos clínicos envolvem métodos menos invasivos, já os tratamentos cirúrgicos exigem métodos mais invasivos. Entre estes, observou-se a indicação, na literatura, da simpatectomia, intervenção cirúrgica que consiste no seccionamento do nervo responsável por estimular a produção de suor, procedimento que objetiva a redução da produção de suor excessivo em áreas como axilas e regiões palmares (CHARELLO E DUTRA, 2018). Destacam também, em casos mais graves, a simpatectomia torácica, para os casos em que os demais métodos de tratamentos apresentarem resposta pouco satisfatória (HAGEMANN E SINIGAGLIA, 2019)

Entre os tratamentos clínicos, foi observada referência aos antitranspirantes à base de cloridrato de alumínio, os quais bloqueiam os canais excretores das glândulas sudoríparas, sendo assim um método não permanente. Houve também referência à iontoforese, um método que utiliza eletricidade leve para desativar temporariamente a glândula sudorípara, e que evidenciou promover significativas melhorias (DOS SANTOS E PAIVA, 2017).

Foi apontada a prescrição de tratamentos que fazem uso de terapêutica medicamentosa, no qual se utilizam anticolinérgicos e betabloqueadores. Conforme o que se aferiu, os anticolinérgicos têm por ação impedir que as glândulas sudoríparas sejam estimuladas à produção de suor, e os betabloqueadores, por sua vez, agem bloqueando a noradrenalina, atuando na diminuição do estresse relacionado à sudorese excessiva (DOS SANTOS E PAIVA, 2017).

O uso de tais tratamentos são medidas clínicas que apresentam relativo sucesso. Porém, trazem consigo significantes efeitos colaterais, como boca seca, tonturas e 
problemas com a micção (CHARELLO E DUTRA, 2018). A literatura analisada evidenciou que existem diversos procedimentos que auxiliam na melhoria dos efeitos ocasionados pela hiperidrose, entretanto, pode ser observado que há quase sempre um contra associado aos benefícios auferidos pelos tratamentos, seja este um gasto excessivo com a manutenção do tratamento, sejam os efeitos colaterais que geram incômodos constantes para o paciente (BERNARDES et al., 2019).

O tratamento utilizando a toxina botulínica tipo $A$, porém, está entre os tratamentos clínicos menos invasivos, de maneira que, pelo que se aferiu, há entendimento de que seja atualmente o tratamento com maior efetividade e segurança para a hiperidrose (HAGEMANN e SINIGAGLIA, 2019).

\subsection{TOXINA BOTULÍNICA E SEU USO NA MEDICINA ESTÉTICA E NA HIPERIDROSE}

A toxina botulínica é a neurotoxina produzida pelo Clostridium botulinum, uma bactéria anaeróbica, que se reproduz em ambientes onde há ausência ou pouca quantidade de oxigênio (CHARELLO E DUTRA, 2018). Há, pelo que se conhece atualmente, sete tipos de toxina botulínica, sendo representadas de $A$ até $G$, pelas letras do alfabeto, sendo que apenas as toxinas dos tipos $A$ e $B$ encontram aplicação na medicina e em outras áreas relacionadas à saúde (HAGEMANN e SINIGAGLIA, 2019).

$\mathrm{Na}$ análise da literatura selecionada, observou-se, em relação ao histórico do uso da toxina botulínica na medicina, o primeiro registro do uso da mesma por Scott e Schantz na década de 70 , tendo sido usada no tratamento do estrabismo (FUJITA E HURTADO, 2018). Nada obstante, a toxina botulínica, embora seja a causadora do botulismo, trata-se da primeira proteína de ordem microbiana a ter sido empregada em tratamentos humanos por meio injetável (GUERRA NETO, 2016).

Atualmente a toxina botulínica é largamente utilizada na medicina e em tratamentos estéticos, sobretudo no campo do rejuvenescimento facial, sendo considerada uma técnica não-invasiva e que evita o recurso a meios cirúrgicos (FUJITA E HURTADO, 2018). Esta popularidade em relação ao largo uso da toxina botulínica em 
procedimentos estéticos pode ser justificado em função da interação bioquímica da mesma, a qual atua nos tecidos do corpo humano promovendo a paralisia dos impulsos neuromusculares que permitem a liberação da acetilcolina, o que acarreta na diminuição da contração muscular (CHARELLO E DUTRA, 2018).

Apesar de possuírem uma alta taxa de toxicidade, sendo uma das toxinas mais letais conhecidas, a toxina botulínica do tipo A têm mecanismos de ação bastante específicos, sendo assim empregadas com segurança em tratamentos de doenças que envolvem espasmos neuromusculares (PINTO; MILAGRES E QUEIROZ, 2015).

Analisou-se que o uso da toxina botulínica na medicina apresenta um largo potencial, sendo utilizada especialmente na medicina estética, podendo ainda ser empregada em áreas diversas como urologia, fisioterapia, oftalmologia, odontologia e outras (FUJITA E HURTADO, 2018). Outrossim, atualmente o BOTOX®, marca produzida pela empresa norte americana Allergan, é considerada a única forma de toxina botulínica tipo A utilizada no tratamento de hiperidrose, como uma opção segura e eficaz (DOS SANTOS E PAIVA, 2017), sendo ainda liberada pela Anvisa (Agência Nacional de Vigilância Sanitária) para o tratamento da hiperidrose e demais procedimentos em que se emprega a toxina botulínica tipo A (BERNARDES et al., 2019).

Em relação ao uso do tratamento da hiperidrose com toxina botulínica tipo $A$, constatase a utilização da neurotoxina como um método seguro, preciso e bem tolerado, e ainda, ficou evidenciado que trata-se de um tratamento de execução rápida, não cirúrgico, e, salvo os casos em que o paciente possui baixíssimo limiar de dor, até mesmo a anestesia se faz dispensável, sendo portanto, praticamente indolor e não agressivo (CHARELLO E DUTRA, 2018).

$\mathrm{Na}$ análise do mecanismo de ação do tratamento, observou-se que a toxina botulínica atua afetando as glândulas sudoríparas, especificamente os nervos simpáticos pósganglionares para as glândulas sudoríparas (GUERRA NETO, 2016). Assim, foi observado na análise dos dado que a neurotoxina causa um bloqueio que, evidentemente pode atrofiar as glândulas sudoríparas mediante o uso prolongado do 
tratamento (HAGEMANN e SINIGAGLIA, 2019). Nessa esteira, a involução provocada pela toxina botulínica, associada à melhora do estado emocional do paciente, com a consequente evolução de sua autoestima, favorece o retardamento da sudorese (PINTO; MILAGRES E QUEIROZ, 2015).

A pesquisa permitiu observar ainda que, como a toxina botulínica impede a liberação da acetilcolina, no tocante à hiperidrose este bloqueio acarreta na inibição do comando que permite a produção de suor (CHARELLO E DUTRA, 2018), este bloqueio ocorre especificamente nas fibras colinérgicas simpáticas pós-ganglionares, evidentemente desabilitando as glândulas sudoríparas écrinas (DOS SANTOS E PAIVA, 2017).

Há, assim, uma neutralização do comando nervoso, o que anula a produção de sudorese. Tal bloqueio, no entanto, não acarreta riscos para o indivíduo, desde que observados os protocolos de segurança, pois o mesmo atua somente na passagem da acetilcolina (PINTO; MILAGRES E QUEIROZ, 2015).

Nesse interim, a pesquisa aqui efetuada também apontou para o fato de que a dosagem ministrada no tratamento é um fator de extrema importância, com alto potencial de inviabilizar o sucesso do tratamento, se a toxina botulínica tipo A for ministrada em quantidades altas, sendo virtualmente um veneno se administrada em doses superiores ao recomendado (PINTO; MILAGRES E QUEIROZ, 2015).

Observou-se que uma dose intravenosa de 0,8 a 0.9 nanogramas de toxina botulínica por quilograma de peso corporal de um indivíduo já pode ser considerada dose letal, e por via oral, uma dose de 0,2 a 1 micrograma já possui o potencial de levar ao óbito (DOS SANTOS E PAIVA, 2017). Deve-se, portanto, ministrar a mínima dosagem necessária, uma vez que a toxina botulínica é uma potente neurotoxina, capaz de causar sequelas irreversíveis, e ainda, e observância à questão da formação de imunidade, sendo a protocolo de administração de suma importância para que não haja aumento antigenicidade (CHARELLO E DUTRA, 2018). 
No tocante à aplicação da toxina botulínica no tratamento da hiperidrose, a análise evidenciou que a aplicação se dá por injeções intradérmicas, de 2,5 a $5 \mathrm{U}$ da toxina, realizadas num padrão de grade, em 45 ou 50 pontos de 1 a $2 \mathrm{~cm}$ de intervalo, com agulhas de pequeno calibre (DOS SANTOS E PAIVA, 2017), devendo tal dose ser ainda, diluída em soro fisiológico $0,9 \%$ e aplicada em intervalos de tempo que variam de 7 a 16 meses (HAGEMANN e SINIGAGLIA, 2019). Diante disso, foi evidenciado também que a repetição excessiva de doses ministradas, bem como a quantidade e o intervalo de tempo entre as ministrações, influi na resposta imunológica do indivíduo (GUERRA NETO, 2016).

A formação involuntária de imunidade à toxina botulínica destaca-se assim como uma das dificuldades do tratamento da hiperidrose com a substância, tendo sido observada tal formação em casos nos quais houve aplicação repetida da dose de toxina botulínica tipo A (FUJITA E HURTADO, 2018), e em casos em que não foi respeitado o período de tempo necessário entre uma aplicação e outra.

Houve indicação na literatura de que doses superiores a 200U, e com intervalos de tempo entre aplicações menores que um mês levaram consequentemente à formação de imunidade no paciente, inviabilizando-se assim o tratamento (GUERRA NETO, 2016). Desta feita, além da obediência ao protocolo de aplicação da menor dose necessária, esta deve ser associada a um período prolongado entre as aplicações, havendo ainda uma relação da quantidade da toxina aplicada por área.

Em relação aos efeitos positivos do tratamento da hiperidrose com a toxina botulínica tipo $A$, verificou-se uma melhora significativa na qualidade de vida dos pacientes submetidos ao procedimento em aproximadamente 7 a 9 meses, devido a diminuição de cerca $75 \%$ na produção de suor (DOS SANTOS E PAIVA, 2017), tendo-se percebido também que houve elevado grau de satisfação no tratamento, com índices baixos de efeitos colaterais e de complicações decorrentes (FUJITA E HURTADO, 2018). No tocante às desvantagens e efeitos colaterais, foram observados efeitos adversos como paralisias reversíveis, fruto da difusão da toxina em decorrência de aplicações mais profundas, e fraqueza. Não obstante, evidenciou-se também em 
alguns casos, equimoses e parestesias, também temporárias e reversíveis (HAGEMANN e SINIGAGLIA, 2019).

Dos Santos e Paiva também aferiram que a administração do BOTOX® no tratamento da hiperidrose primária acarreta como efeitos adversos, dores na aplicação, incômodos que desaparecem em poucos dias e hematomas (DOS SANTOS E PAIVA, 2017). Observou-se, com peculiaridade, que o tratamento da toxina botulínica não é indicado para indivíduos que estejam com a saúde comprometida, no que se refere à imunidade e ao quadro neuromuscular. Entre os grupos contraindicados, encontramse ainda gestantes e lactantes (GUERRA NETO, 2016).

$\mathrm{Na}$ análise das vantagens do uso da toxina botulínica tipo A no tratamento da hiperidrose em relação aos outros disponíveis, o que se observou foi, primeiramente, uma vantagem relacionada ao custo-benefício (HAGEMANN e SINIGAGLIA, 2019), e em seguida, em relação ao conforto do paciente, uma vez que é um tratamento que apresenta pouco potencial invasivo e complicações raras e reversíveis (CHARELLO E DUTRA, 2018).

\section{CONSIDERAÇÕES FINAIS}

A toxina botulínica se mostrou uma das substâncias mais eficazes e de melhor custobenefício para o tratamento da hiperidrose. Trata-se de uma técnica que, se devidamente empregada, apresenta baixo potencial de lesividade para o paciente, pois seus efeitos colaterais são mínimos e reversíveis, e sua utilização não é invasiva. Ainda, há que se observar uma maior popularização do tratamento, a fim de baratear o mesmo e promover o acesso de indivíduos acometidos pela doença. $O$ tratamento da hiperidrose utilizando a toxina botulínica promove uma melhoria da qualidade de vida, na medida em que permite ao paciente transpor fobias sociais e situações constrangedoras, acarretando uma melhoria no quadro psicoemocional, sem necessariamente precisar submetê-lo a um procedimento cirúrgico ou invasivo. 


\section{REFERÊNCIAS}

BASTOS, Aline de Carvalho et al. Impacto social da hiperidrose primária: revisão de literatura. Semana de Pesquisa da Universidade Tiradentes-SEMPESq, n. 18, 2018.

BERNARDES, Nicole Blanco et al. Estudo Sistematizado consoante a excelência na terapêutica com Toxina Botulínica do Tipo A em pacientes com Quadros de Hiperidrose. ID on line REVISTA DE PSICOLOGIA, v. 13, n. 45, p. 203-219, 2019.

CHARELLO, Daniely de Souza; DUTRA, Robertson. 0 uso da toxina botulínica no tratamento da hiperidrose palmar e axilar. Revista Eletrônica Biociências, Biotecnologia E Saúde, v. 12, n. 24, p. 17-29, 2019.

DOS SANTOS, Camila Z. P.; PAIVA Letícia M. Efeitos da toxina botulínica tipo a no tratamento da hiperidrose primária. 2017. 21 f. Monografia (Graduação) Faculdade de Ciências da Educação e Saúde, Centro Universitário de Brasília, Brasília, 2017.

FUJITA, Rita L. R.; HURTADO, Carola C. N. Aspectos relevantes do uso da toxina botulínica no tratamento estético e seus diversos mecanismos de ação. Saber Científico, Porto Velho, V., n., p. - , mês./mês. 2018.

GUERRA NETO, Pedro Gonçalves da Silva. Toxina Botulínica Tipo A: ações farmacológicas e riscos de uso nos procedimentos estéticos faciais. 2016. 44f. Monografia (Graduação) - Instituto Nacional de Ensino Superior e Pesquisa e Centro de Capacitação Educacional, Recife, 2016.

HAGEMANN, Daniela; SINIGAGLIA, Giovana. Hiperidrose e o uso da toxina botulínica como tratamento: revisão bibliográfica. Revista Destaques Acadêmicos, v. 11, n. 3, 2019. 
PINTO, Bianca L. L.; MILAGRES, Bruno S.; QUEIROZ, Lorena B. A toxina botulínica tipo A como tratamento para hiperidrose primária. 2015. $18 \mathrm{f}$. Monografia (Graduação) - Faculdade de Ciências da Educação e Saúde, Brasília, 2015.

Enviado: Outubro, 2020.

Aprovado: Outubro, 2020. 